\title{
Almuth Grésillon, La Mise en œuvre. Itinéraires génétiques, Paris, CNRS Éditions, 2008, 305 p.
}

\section{Estanislao Sofía}

\section{(2) OpenEdition}

1 Journals

\section{Édition électronique}

URL : http://journals.openedition.org/genesis/664

DOI : $10.4000 /$ genesis. 664

ISSN : 2268-1590

Éditeur :

Presses universitaires de Paris Sorbonne (PUPS), Société internationale de génétique artistique littéraire et scientifique (SIGALES)

Édition imprimée

Date de publication : 30 octobre 2011

Pagination : 194-195

ISBN : 978-2-84050-804-5

ISSN : 1167-5101

\section{Référence électronique}

Estanislao Sofía, « Almuth Grésillon, La Mise en œuvre. Itinéraires génétiques, Paris, CNRS Éditions, 2008, 305 p. », Genesis [En ligne], 33 | 2011, mis en ligne le 23 octobre 2013, consulté le 22 septembre 2020. URL : http://journals.openedition.org/genesis/664; DOI : https://doi.org/10.4000/genesis.664 
Les six études regroupées dans la troisième partie, «La traduction littéraire : en quête de l'équivalence », sont axées sur un autre type d'adaptation de la culture étrangère. Lidiïa Sazonova décrit les modes d'assimilation du langage courtois par la littérature russe ; Elena Gretchanaïa retrace la genèse de la traduction, par Vassili Trediakovski, du Voyage de l'île d'Amour ; Andreï Mikhaïlov étudie de quelle manière Prosper Mérimée a traduit La Dame de pique de Pouchkine ; Nina Koroliova présente l'activité de traduction d'Anna Akhmatova ; Caroline Bérenger examine les écrits en français de Marina Tsvetaeva ; Tamara Balachova traite de l'interaction des langues dans la version russe de pièces de Lope de Vega.

Les auteurs des cinq articles de la quatrième partie, « Le plurilinguisme dans les écrits non littéraires », cherchent à comprendre les processus d'interférence entre les langues dans des textes non fictionnels. Dans le Journal du voyage en Italie de Michel de Montaigne, Kirill Tchekalov analyse l'interaction du latin, du français et de l'italien ; Catherine Viollet observe les pratiques et fonctions du multilinguisme dans des journaux intimes rédigés en français par des Russes ; Sylvie Courtine-Denamy réfléchit sur le plurilinguisme dans le Journal de pensée d'Hannah Arendt ; dans l'article de Nadejda Podzemskaia, il s'agit de saisir à quoi correspond la superposition des langues dans les écrits théoriques de Vassily Kandinsky ; Vadim Polonski se donne pour objet d'analyse les jeux langagiers plurilingues dans la correspondance de Dmitriï Merejkovski.

La dernière partie, intitulée « Une autre langue dans les manuscrits monolingues », comprend trois articles qui sont consacrés, respectivement, aux éléments linguistiques « étrangers » dans des manuscrits de Vladimir Maïakovski (Vera Tériokhina), d'Igor Severianine (Natalia ChoubnikovaGousseva) et de Romain Gary (Valentina Chepiga).
Le présent recueil d'articles montre quel vaste champ de recherches ouvrent les études génétiques de textes multilingues, aussi bien du point de vue des généralisations théoriques que des travaux ponctuels sur tel ou tel manuscrit. Différents types de manuscrits peuvent être concernés : ceux qui ont été réalisés dans un milieu social multilingue, en langue étrangère ou avec des fragments de taille variable en langue étrangère, mais aussi les brouillons des traducteurs. Sur le plan des réflexions théoriques, les participants au symposium ont produit un grand nombre d'observations, et ce à partir d'approches diverses (grammaticales, stylistiques, thématiques, fonctionnelles, etc.), même si, comme le remarquent Pierre-Marc de Biasi, Olga Anokhina et Natalia Velikanova, l'étude des problèmes théoriques du multilinguisme dans la genèse du texte ne fait que commencer.

\section{Almuth Grésillon, La Mise en auvre. Itinéraires génétiques, Paris, CNRS Éditions, 2008, 305 p.}

\section{Compte rendu par Estanislao Sofia}

Ce volume réunit, soit abrégés, soit étoffés, treize articles publiés par l'auteur sur une période de presque vingt ans (1988-2007). Frappent, lorsqu'on considère cette ampleur, la cohérence et la systématicité avec lesquelles ont été agencés les contenus. À part une « Ouverture » et une « Coda », la table des matières comporte trois parties. La première, théorique (p. 19-96), retrace les origines et les fondements de la critique génétique, et constitue en ce sens une suite de l'ouvrage (phare) publié par l'auteur en 1994, Éléments de critique génétique (PUF). La deuxième partie (p. 99-241), plutôt pratique, est une mise en æeuvre des principes et des méthodes décrits dans la première, d'où le titre de l'ouvrage. Cinq dossiers jalonnent cette démarche, centrée tour à tour sur Flaubert, Zola,
Proust, Supervielle et Francis Ponge. En fin de volume, deux études se partagent la troisième partie (p. 245-294), qui explore, en guise de clôture, les « frontières » de la critique génétique.

L'« ouverture » sert d'introduction aux problématiques et principes mis en relief par la critique génétique. Elle se donne pour objet de revendiquer le caractère autonome de la discipline, tout en soulignant la pluralité de systèmes conceptuels (narratologique, psychanalytique, linguistique, poétique, etc.) susceptibles d'encadrer sa production.

La première partie, continuant le fil théorique de l'ouverture, insiste sur les méthodes essentielles et nécessaires au décodage des manuscrits modernes. Parmi les développements les plus intéressants, on y trouve une mise en place de la différence (radicale) entre "brouillon », « manuscrit» et « texte» (chap. I et IV), ainsi que l'examen des procédés à suivre pour déchiffrer les premiers (chap. III et $\mathrm{V}$ ) afin de pouvoir les exploiter dans l'établissement de la genèse du dernier (chap. II). L'auteur inscrit la discipline au nombre des approches « majeures » du phénomène littéraire : c'est ce qui sera exemplifié dans la deuxième partie.

Le dossier de Hérodias, de Flaubert, permet de souligner (p. 101) que les manuscrits «parlent souvent un langage plus explicite que le texte imprimé » (je souligne), ou qu'ils montrent «plus clairement que le texte imprimé » ce qu'il y a à voir dans ce dernier (p. 127). Les manuscrits offrent ainsi au généticien un terrain supplémentaire pour interpréter l'œuvre analysée, plutôt que pour établir sa genèse. L'objectif de l'analyse génétique est ici de faire voir le « diffus » (p. 101) qui n'est pas dans l'imprimé, afin d'enrichir celui-ci. On suit ainsi l'auteur dans sa quête "d'autres traits », " plus nombreux et plus diversifiés dans l'avanttexte que dans le texte imprimé » (p. 122), qui « confirment», par exemple, «l'aspect 
irréel et dématérialisé de Salomé ». L'œuvre est ainsi étoffée par le matériau trouvé dans les manuscrits. Le dossier nous semble une lecture critique du texte flaubertien à l'aide des manuscrits plutôt qu'une étude prioritairement génétique.

Tout autre est le cas du dossier Zola, représentatif en tout point de ce qu'on s'était habitué à attendre des développements de la discipline. Des indices matériels échelonnés sur une pluralité de phases rédactionnelles révèlent les modalités de création propres à cet auteur, attachées ici aux vicissitudes du style avant-textuel, elles-mêmes centrées sur la genèse du dénouement de La Bête humaine. On apprécie l'adresse avec laquelle Almuth Grésillon illustre, en parcourant des documents avant-textuels, les hasards de la gravitation autour de ces trois pôles classiques guidant ensemble la rédaction : inventio, dispositio, elocutio, mêlés chez Zola aux trois pôles ayant fédéré ses sources d'inspiration : expérience vécue, documentation " scientifique », imaginaire romanesque. $\mathrm{Au}$ nombre des issues inhérentes à ce parcours, on remarque la mise en relief de cette modalité d'invention " tâtonnante » qu'on avait jadis voulu exclure des habitudes de cet écrivain. Celui qui avait été regardé comme l'écrivain prototypique de «l'écriture à programme » se réservait donc, lui aussi, le droit d'improviser

C'est à Proust qu'est consacrée la troisième étude, également illustrative de la force analytique de la démarche génétique. C'est la découverte de ces mouvements d'écriture propres à chaque écrivain qui fait de la critique génétique une discipline digne d'attention. On apprend que dans le « vaste processus scriptural », chez Proust, jamais rien ne se perd, dans cette sorte de « transformation ininterrompue » qui devait donner à l'œuvre proustienne l'unité que l'on sait. L'élément choisi pour le montrer ? Le thème de « la matinée », dont on suit la genèse depuis les premières ébauches du Contre Sainte-Beuve, à travers les manuscrits des différentes parties de la Recherche, jusqu'à La Prisonnière. Exemple d'étude génétique « verticale », axée ici sur la genèse d'un thème et son évolution à travers une œuvre en devenir.

Les deux dernières études, consacrées à la genèse d'un poème, étaient par cela même marquées du sceau du risque : peut-on " expliquer » la genèse d'un poème ? Ce n'est pas la question que se pose l'auteur, mais plutôt : «Que nous apprend la genèse de ce [type de] texte sur les rapports complexes entre sens, sons et formes poétiques ?» (p. 179). Le parcours à travers les différentes étapes de la constitution de Vivre encore de Supervielle débouche sur une conclusion intéressante : le poème, dont on pourra éventuellement retrouver les traces des sources d'inspiration, n'est finalement qu'un produit dû aux alternatives bifurquées (on a envie de dire aux caprices) de la plume et de l'esprit, aux fourvoiements, dirait-on même, de la raison. Souligner ces aspects erratiques inhérents à la genèse poétique préserve en même temps du "péché téléologique » qui n'est qu'une sorte de « marche inexorable du poème vers sa forme parfaite ». La forme finale du poème, parfaite ou imparfaite, serait elle aussi traversée, paradoxalement, par des chemins qui n'y convergent pas.

L'analyse de L'Ardoise de Francis Ponge confirme ce résultat, en y ajoutant cependant des précisions sur les modes de documentation propres au travail de ce poète. Parmi les plus remarquables : l'utilisation récurrente des dictionnaires, dont les définitions servent de déclencheurs de « l'association la plus libre » donnant lieu à une «rêverie lexicale » qui ne fait que contraster avec la genèse de la syntaxe, qui serait, elle, « étonnamment monotone ». Que toute référence intertextuelle soit effacée au profit d'un simulacre de clôture, de « discrétion », de forme « épurée et elliptique », ne montre que mieux la valeur de l'analyse génétique, visant à dévoiler, sous ce masque de pureté, l'aspect laborieux, instable et bien souvent furtif du travail de création. D'où la conclusion, valable pour les cinq dossiers étudiés, selon laquelle « il n'y a[urait] donc plus une seule forme définitivement arrêtée ; par essence, elle est toujours amendable, révisable, multipliable, cumulable, transformable » (p. 238).

Avait-on besoin de la critique génétique pour s'en persuader ? Peut-être pas. Mais cette discipline le montre mieux que d'autres méthodes : c'est ce qui fait toute la valeur du volume présenté par Almuth Grésillon.

Les deux études exploratoires qui se partagent la troisième partie soumettent bel et bien la discipline $a ̀$ l'épreuve. La première, consacrée aux spécificités de la genèse théâtrale, dévoile l'hétérogénéité fondamentale de cette dernière par rapport aux genèses des genres traditionnellement étudiés par les généticiens. La définition et le statut de l'œuvre s'avèrent problématiques dans la mesure où son écriture, régie par des principes parfois discordants du texte et de la scène, et bien des fois confiée à deux ou plusieurs « mains », fait éclater la notion d'auteur en une pluralité qui trouve partout à se répercuter. L'absence de «bon à tirer », les remaniements continuels à chaque nouvelle mise en scène obligent entre autres à une réélaboration des notions traditionnelles de « texte », « auteur », « fin ». La dernière étude («Une critique génétique sans brouillons ? ») problématise, elle, les notions de «brouillon » et de « manuscrit ». C'est donc finalement la totalité des concepts génétiques (ou presque) qui sont mis en question. Conclusion : si la critique génétique est vouée à perdurer, elle devra s'atteler à un travail de redéfinition des concepts qui ont constitué ses fondements. Travail que l'ouvrage d'Almuth Grésillon aborde, via la mise en œuvre et à l'épreuve de sa discipline, de manière exemplaire. 